\title{
Coupling spans of the Higgs-like boson
}

\section{Bogdan A. Dobrescu and Joseph D. Lykken}

Theoretical Physics Department, Fermilab, P.O. Box 500, Batavia, IL 60510, U.S.A.

E-mail: bdob@fnal.gov, lykken@fnal.gov

Abstract: Using the LHC and Tevatron data, we set upper and lower limits on the total width of the Higgs-like boson. The upper limit is based on the well-motivated assumption that the Higgs coupling to a $W$ or $Z$ pair is not much larger than in the Standard Model. These width limits allow us to convert the rate measurements into ranges for the Higgs couplings to various particles. A corollary of the upper limit on the total width is an upper limit on the branching fraction of exotic Higgs decays. Currently, this limit is $47 \%$ at the 95\% CL if the electroweak symmetry is broken only by doublets.

Keywords: Higgs Physics, Beyond Standard Model

ArXiv EPRINT: 1210.3342 


\section{Contents}

1 Introduction $\quad 1$

2 Measurable quantities $\quad 2$

3 Limits on the total Higgs width 5

3.1 Upper limit on $\Gamma_{h} \quad 5$

$\begin{array}{lll}3.2 & \text { Lower limit on } \Gamma_{h} & 7\end{array}$

4 Limits on Higgs couplings and non-standard decays $\quad 9$

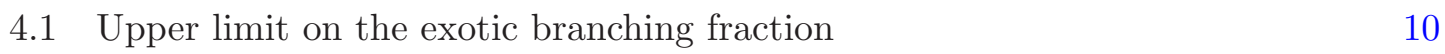

$\begin{array}{lll}4.2 & \text { Spans of the Higgs couplings } & 10\end{array}$

5 Conclusions $\quad 12$

\section{Introduction}

The discovery of a Higgs-like particle $\left(h^{0}\right)$ in the $\gamma \gamma$ and $4 \ell$ final states by the ATLAS [1] and CMS [2] Collaborations, of mass $M_{h^{0}}$ roughly in the $125-126.5 \mathrm{GeV}$ range, provides multiple opportunities for probing phenomena beyond the Standard Model (SM). The SM Higgs boson [3] in that mass range has a very small total width, $\Gamma_{h}^{\mathrm{SM}} / M_{h}=3.2 \times 10^{-5}$, due to the small Yukawa coupling of the $b$ quark $\left(y_{b} \sim 0.02\right)$ and the severe phase-space suppression of the $W W^{*}$ final state. Therefore, if new particles lighter than about $60 \mathrm{GeV}$ have a coupling to the Higgs doublet larger than $10^{-2}$, the Higgs-like boson can have a large branching fraction $\mathcal{B}_{X}$ into exotic final states, and consequently a larger total width, $\Gamma_{h}>\Gamma_{h}^{\mathrm{SM}}$. The exotic Higgs decays could escape detection for a long time, for example in the case of the four gluon-jet final state arising from $h \rightarrow A^{0} A^{0} \rightarrow 4 g$ where $A^{0}$ is a gauge-singlet spin-0 particle [4-6].

Thus, it is important to analyze whether a relatively large $\Gamma_{h}$ can be observable. The prospects for measuring the line shape of $h^{0}$ are rather dim, barring a high-luminosity muon collider running at $\sqrt{s}=M_{h}$. Nevertheless, one may hope to determine $\Gamma_{h}$ indirectly, given that all the rates for Higgs signals at colliders are inversely proportional to $\Gamma_{h}$. It turns out, however, that the effect on rates of a larger $\Gamma_{h}$ can be compensated by an universal increase of the $h^{0}$ couplings. In fact, at hadron colliders the only observables based on rates are a product of the squared couplings for producing and decaying $h^{0}$ divided by $\Gamma_{h}$, so that the width itself cannot be measured even indirectly at the LHC. ${ }^{1}$

\footnotetext{
${ }^{1}$ At $e^{+} e^{-}$or $\mu^{+} \mu^{-}$colliders the recoil of the $Z$ produced in association with $h^{0}$ would allow a measurement of the $h^{0} Z Z$ coupling, and consequently $\Gamma_{h}$ can be determined from the rates for various processes.
} 
The impossibility of measuring $\Gamma_{h}$ at the LHC hampers the extraction of Higgs couplings from the rate measurements. In order to go around this problem, several groups have relied on assumptions about the width. For example, it has been assumed that only SM decays are allowed [7-13], or that all nonstandard final states include particles escaping the detector [14-16], or that nonstandard final states are allowed only when certain couplings to SM particles are the same as in the SM [17-22]. A more general framework is allowed in [23], but the problem of rescaling both the width and the couplings is avoided by imposing an ad-hoc upper limit on some of the couplings.

In this paper we use the ATLAS and CMS rate measurements to derive an upper limit on $\Gamma_{h}$ based on the rather robust theoretical assumption that the Higgs coupling to a $W$ pair is not much larger than in the SM. ${ }^{2}$ In many models, the $h^{0} W W$ coupling is substantially smaller than in the SM, and only in the unusual case [25-30] of large VEVs for higher $\mathrm{SU}(2)_{W}$ representations does the coupling exceed its $\mathrm{SM}$ value. ${ }^{3}$ We then translate this limit on $\Gamma_{h}$ into an upper limit on the exotic branching fraction, $\mathcal{B}_{X}$. Furthermore, we derive a lower limit on $\Gamma_{h}$ from the Tevatron [31] and LHC [1, 2] rate measurements, especially for the $b \bar{b}$ and $W W^{*}$ decay modes. Having bounded $\Gamma_{h}$ from above and below, we can then extract nearly-model independent upper and lower limits on the general couplings of the Higgs-like particle allowed by various rate measurements. Our method of deriving the spans of the couplings could be used by the CMS and ATLAS Collaborations in order to translate their measurements into information about the couplings of the Higgs-like particle in a way that is as model-independent as possible at hadron colliders.

In section 2 we parametrize the general couplings of the Higgs-like boson to SM particles, and summarize the existing rate measurements. The upper and lower limits on $\Gamma_{h}$ are derived in section 3 . In section 4 we compute the upper limit on the branching fraction of exotic Higgs decays, and then we obtain the coupling spans. Our conclusions are included in section 5 .

\section{Measurable quantities}

A Higgs boson is a scalar particle $h^{0}$ that couples to the $W$ and $Z$ bosons according to

$$
\frac{g}{M_{W}} h^{0}\left(C_{W} M_{W}^{2} W^{+} W^{-}+C_{Z} \frac{M_{Z}^{2}}{2} Z Z\right)
$$

where $g$ is the $\mathrm{SU}(2)_{W}$ gauge coupling, and $C_{W}$ and $C_{Z}$ parametrize the deviation from the SM couplings: $C_{W}^{\mathrm{SM}}=C_{Z}^{\mathrm{SM}}=1$. If electroweak symmetry breaking is due entirely to VEVs of $\mathrm{SU}(2)_{W}$ doublets, then $[9,14,32]$

$$
0<C_{W}=C_{Z} \leq 1 \text { for doublet VEVs. }
$$

In models where triplets or higher $\mathrm{SU}(2)_{W}$ representations acquire VEVs it is possible to have $C_{W} \neq C_{Z}$ as well as values for $C_{W}$ and/or $C_{Z}$ above 1 or negative [26]. However,

\footnotetext{
${ }^{2}$ This procedure was mentioned in refs. $[9,14,24]$ but was not explored in detail in the general case where all couplings are free parameters and nonstandard decay modes are allowed.

${ }^{3} \mathrm{An} h^{0} W W$ coupling larger than in the SM also leads to unitarity violation in longitudinal $W W$ scattering unless there are isospin-2 resonances [30].
} 
such models predict additional scalars, including doubly-charged and singly-charged particles, whose effects are tightly constrained by the electroweak data [33] and by collider searches [34]. As a result, one can still derive some upper bounds on the couplings:

$$
\left|C_{W}\right|<C_{W}^{\max }, \quad\left|C_{Z}\right|<C_{Z}^{\max } .
$$

with $C_{W}^{\max }, C_{Z}^{\max }=O(1)$.

For example, the Georgi-Machacek model [25] includes a real triplet and a complex triplet (such that custodial invariance may arise due to a cancellation between the contributions of the two triplets), and also a complex doublet whose VEV is necessary for giving the top mass. Due to the loop contributions of charged scalars to the $Z b \bar{b}$ vertex [33], the deviations from the SM couplings have upper limits $C_{W}^{\max }, C_{Z}^{\max } \approx 1.5[26]$.

The couplings of a Higgs boson to third generation fermions can be written as

$$
-C_{t} \frac{m_{t}}{v_{h}} h^{0} \bar{t} t-C_{b} \frac{m_{b}}{v_{h}} h^{0} \bar{b} b-C_{\tau} \frac{m_{\tau}}{v_{h}} h^{0} \bar{\tau} \tau
$$

where $m_{t}, m_{b}$ and $m_{\tau}$ are the $t, b$ and $\tau$ masses, $v_{h} \approx 246 \mathrm{GeV}$ is the electroweak scale, and $C_{t}, C_{b}, C_{\tau}$ are real parameters that are equal to 1 in the SM.

The Higgs boson coupling to the top quark, and possibly to new colored particles, induces a 1-loop coupling of $h^{0}$ to a pair of gluons. In the approximation where $M_{h} / m_{t}$ effects are negligible (they turn out to be below $7 \%$ for $M_{h} \approx 125 \mathrm{GeV}$ ) and where new colored particles that couple to $h^{0}$ can be integrated out, the Higgs coupling to a pair of gluons is given by a dimension- 5 operator:

$$
C_{g} \frac{\alpha_{s}}{12 \pi v_{h}} h^{0} G^{\mu \nu} G_{\mu \nu},
$$

where $G^{\mu \nu}$ is the gluon field strength, and $C_{g}$ is a real parameter (equal to 1 in the SM). If there are new colored particles with mass not much larger than $M_{h}$ that couple to $h^{0}$ (such as a color-octet scalar [35-38]), then $C_{g}$ should be replaced by a function of $M_{h}$.

The only other Higgs couplings relevant here involve photons and arise also at one loop. These lead to the $h^{0} \rightarrow \gamma \gamma, Z \gamma$ decays. Given that the dominant contributions to these decays in the SM arise from $W$ loops [3], it is not accurate to parametrize these couplings by the dimension-5 operators $h^{0} F^{\mu \nu} F_{\mu \nu}$ and $h^{0} Z^{\mu \nu} F_{\mu \nu}$. For example, in the SM the full 1-loop $\Gamma^{\mathrm{SM}}\left(h^{0} \rightarrow \gamma \gamma\right)$ width is $50 \%$ larger than the result based on the dimension-5 operator for $M_{H} \approx 125 \mathrm{GeV}$. We are thus led to define the deviations from the SM effective couplings to photons by

$$
\begin{aligned}
C_{\gamma} & \equiv\left(\frac{\Gamma\left(h^{0} \rightarrow \gamma \gamma\right)}{\Gamma^{\mathrm{SM}}\left(h^{0} \rightarrow \gamma \gamma\right)}\right)^{1 / 2}, \\
C_{Z \gamma} & \equiv\left(\frac{\Gamma\left(h^{0} \rightarrow Z \gamma\right)}{\Gamma^{\mathrm{SM}}\left(h^{0} \rightarrow Z \gamma\right)}\right)^{1 / 2}
\end{aligned}
$$

There are several processes at hadron colliders that can be studied in order to determine the couplings shown in eqs. (2.1), (2.4), (2.5) and (2.6). Higgs production proceeds 
mainly through gluon fusion, vector boson fusion (VBF), $W h^{0}$ or $Z h^{0}$ associated production, or radiation off a top quark $\left(t \bar{t} h^{0}\right)$. The cross sections for these five processes are proportional to $C_{g}^{2},\left(C_{W}^{2}+r C_{Z}^{2}\right), C_{W}^{2}, C_{Z}^{2}$, and $C_{t}^{2}$, respectively. The parameter $r$ that sets the ratio of rates for $Z Z$ to $W W$ fusion is typically between 0.3 and 0.5 in $p p$ collisions, and depends on the center-of-mass energy [39].

The widths for the Higgs decays to $b \bar{b}, \tau^{+} \tau^{-}, W W, Z Z, \gamma \gamma, Z \gamma$, are proportional to $C_{\mathcal{P}}^{2}$ where $\mathcal{P}=b, \tau, W, Z, \gamma, Z \gamma$, respectively. Additional decay modes, to final states involving SM particles (e.g., $h^{0} \rightarrow A^{0} A^{0} \rightarrow 4 j$ where $A^{0}$ is a new spin-0 particle [4-6]), or new stable particles may have large contributions to $\Gamma_{h}$.

The narrow width approximation is accurate for $M_{h} \approx 125 \mathrm{GeV}$ even if new physics contributions to $\Gamma_{h}$ were three orders of magnitude larger than $\Gamma_{h}^{\mathrm{SM}}$, the total Higgs width in the SM. Thus, the cross section for a process of Higgs production and decay is proportional to

$$
\frac{C_{\text {prod. }}^{2} C_{\text {decay }}^{2}}{\Gamma_{h}},
$$

where $C_{\text {prod. }}$ and $C_{\text {decay }}$ are the $C_{\mathcal{P}}$ coefficients entering the production and decay, respectively, as discussed above. It is convenient to define the "apparent squared-couplings"

$$
a_{\mathcal{P}} \equiv C_{\mathcal{P}}^{2}\left(\frac{\Gamma_{h}^{\mathrm{SM}}}{\Gamma_{h}}\right)^{1 / 2}, \quad \text { for } \mathcal{P}=W, Z, g, \gamma, Z \gamma, t, b, \tau,
$$

so that the cross section for a Higgs process [proportional to the quantity in eq. (2.7)] over the SM cross section for the same process is simply a product of two $a_{\mathcal{P}}$ 's.

The measurements of various Higgs processes allows the determination of the $a_{\mathcal{P}}$ quantities. For example, $a_{b}$ and $a_{W}$ may be extracted from the measured total cross sections for $p p \rightarrow W^{*} \rightarrow W h^{0}$ followed by $h^{0} \rightarrow b \bar{b}$ or $h^{0} \rightarrow W^{+} W^{-}$, through the relations:

$$
\begin{gathered}
\left(\frac{\sigma}{\sigma_{\mathrm{SM}}}\right)(W h \rightarrow W b \bar{b})=a_{W} a_{b}, \\
\left(\frac{\sigma}{\sigma_{\mathrm{SM}}}\right)(W h \rightarrow W W W)=a_{W}^{2},
\end{gathered}
$$

where $\sigma$ is the measured cross section and $\sigma_{\mathrm{SM}}$ is its theoretical value in the SM. Likewise, measurements of the cross sections for various Higgs production mechanisms followed by various Higgs decays determine other products of $a_{\mathcal{P}}$ 's, as shown in table 1.

A fit to the measured cross sections listed in table 1 can determine $a_{\mathcal{P}}$ for $\mathcal{P}=$ $b, W, Z, g, \tau, \gamma$. Only channels that are already measured ${ }^{4}$ or will be probed in the near future are included in table 1. Many other channels such as $g g \rightarrow h^{0} \rightarrow Z \gamma$ (proportional to $\left.a_{g} a_{Z \gamma}\right), W h^{0}$ production followed by $h^{0} \rightarrow Z Z^{*}$ (proportional to $a_{W} a_{Z}$ ), $Z h^{0}$

\footnotetext{
${ }^{4}$ We extract the signal strength for different channels reported by the CMS collaboration from ref. [41], figure 10, right panel. Due to the poor mass resolution of the $W W^{*}, b \bar{b}$ and $\tau^{+} \tau^{-}$channels, the combination with the ATLAS data for $M_{h}=126 \mathrm{GeV}$ should be rather insensitive to the mass difference. We extract the signal strength reported by the ATLAS collaboration for the $4 \ell$ channel from ref. [42], figure 16a for $M_{h}=125 \mathrm{GeV}$, and for the $\gamma \gamma$ channels from ref. [43], figure $14 \mathrm{c}$, which assumes $M_{h}=126.5 \mathrm{GeV}$. The combination with the CMS data for $M_{h}=125 \mathrm{GeV}$ is warranted under the assumption that the $\gamma \gamma$ mass peaks will migrate toward each other.
} 
production followed by $h^{0} \rightarrow \tau \tau$ (proportional to $a_{Z} a_{\tau}$ ), or $t \bar{t} h$ production followed by $h^{0} \rightarrow W^{+} W^{-}, Z Z, \tau^{+} \tau^{-}$, will likely require a sizable integrated luminosity due to their small rates or large backgrounds.

The measurements on the rows labelled by $g g \rightarrow h^{0}$ are dominated by gluon fusion but also contain some contributions of order $10 \%$ from VBF and from associated production with a $W$ or $Z$ decaying hadronically. For simplicity we neglect those contributions.

The measurements on the rows labeled by VBF include two forward jets. The selections used make VBF the dominant production mechanism, but do not eliminate completely the gluon fusion mechanism with two additional jets (simulations within the SM show that this contamination is about $30 \%$ [2]). This effect will convolute the determination of the $a_{g}, a_{W}$, and $a_{Z}$ parameters, but taking it into account is beyond the scope of this article.

\section{Limits on the total Higgs width}

In this section we derive the lower and upper limits on the total width $\Gamma_{h}$ of $h^{0}$.

\subsection{Upper limit on $\Gamma_{h}$}

The existence of a stringent upper limit $\Gamma_{h}^{\max }$ (with $\Gamma_{h}^{\max } \ll M_{h}$ ) on the total $h^{0}$ width is not obvious. After all, the observable quantities $a_{\mathcal{P}}$ can be kept fixed when $\Gamma_{h}$ is increased by increasing all the couplings $C_{\mathcal{P}}$. The reason that there is a useful upper limit stems from the fact that there are upper limits on $C_{W}$ and $C_{Z}$, which are related to the $W$ and $Z$ masses.

Once the $a_{W}$ and $a_{Z}$ quantities are extracted from a fit to the data, each of the upper limits on the Higgs couplings to $W W$ and $Z Z$ gives an upper limit on $\Gamma_{h}$. Using the upper limits on $C_{W}$ and $C_{Z}$ as parametrized in eq. (2.3), we find that eq. (2.8) implies the following upper limit on the total $h^{0}$ width:

$$
\Gamma_{h} \leq \Gamma_{h}^{\max }=\operatorname{Min}\left\{\frac{\left(C_{W}^{\max }\right)^{4}}{a_{W}^{2}}, \frac{\left(C_{Z}^{\max }\right)^{4}}{a_{Z}^{2}}\right\} \Gamma_{h}^{\mathrm{SM}} .
$$

Note that this upper limit relies solely on the existence of an upper limit on the Higgs couplings to $W$ or $Z$; for example, it allows any contribution to the width from exotic Higgs decays.

In the case where the electroweak symmetry is broken only by the VEVs of $\mathrm{SU}(2)_{W}$ doublets (which covers the majority of theories discussed in the literature), the upper limit takes the form

$$
\Gamma_{h} \leq \Gamma_{h}^{\max }=\frac{\Gamma_{h}^{\mathrm{SM}}}{a_{V}^{2}},
$$

where $a_{V}$ is now obtained by the fit performed with the $a_{W}=a_{Z} \equiv a_{V}$ constraint. Note that $a_{V}$ can be measured directly from VBF or associated $V h^{0}$ production followed by $h^{0} \rightarrow W W$ or $Z Z$. The experimental uncertainties in these channels are too large for now, so that we use a more indirect method for extracting $a_{V}$.

Let us first combine the $g g \rightarrow h^{0} \rightarrow W W^{*}$ and $g g \rightarrow h^{0} \rightarrow Z Z^{*}$ rate measurements shown in table 1 , using the prescription of ref. [44, 45] for asymmetric errors:

$$
\left(\sigma / \sigma_{\mathrm{SM}}\right)\left(g g \rightarrow h \rightarrow V V^{*}\right)=0.96_{-0.24}^{+0.27},
$$




\begin{tabular}{|c|c|c|c|}
\hline$h^{0}$ decay & $h^{0}$ production & observable & measured $\sigma / \sigma_{\mathrm{SM}} ; \quad M_{h}=125 \mathrm{GeV}$ \\
\hline \multirow{4}{*}{$W W^{*}$} & $g g \rightarrow h^{0}$ & $a_{g} a_{W}$ & $\begin{array}{l}1.3 \pm 0.5, \text { ATLAS }[1] ; 126 \mathrm{GeV} \\
0.6_{-0.4}^{+0.5}, \text { CMS }[2] ; 125.5 \mathrm{GeV} \\
0.3_{-0.3}^{+0.8}, \text { Tevatron }[40] \\
\text { our average: } 0.9 \pm 0.4\end{array}$ \\
\hline & VBF & $\left(a_{W}+r a_{Z}\right) /(1+r) a_{W}$ & $0.3_{-1.6}^{+1.5}, \mathrm{CMS}[41]$ \\
\hline & $W^{*} \rightarrow W h^{0}$ & $a_{W}^{2}$ & $-2.9_{-2.9}^{+3.2}$, CMS [41] \\
\hline & $Z^{*} \rightarrow Z h^{0}$ & $a_{Z} a_{W}$ & \\
\hline \multirow[t]{2}{*}{$Z Z^{*}$} & $g g \rightarrow h^{0}$ & $a_{g} a_{Z}$ & $\begin{array}{l}1.3_{-0.5}^{+0.7}, \text { ATLAS }[42] \\
0.7_{-0.4}^{+0.5}, \text { CMS }[2] ; 125.5 \mathrm{GeV} \\
\text { our average: } 1.0_{-0.3}^{+0.4}\end{array}$ \\
\hline & $\mathrm{VBF}$ & $\left(a_{W}+r a_{Z}\right) /(1+r) a_{Z}$ & \\
\hline \multirow[t]{2}{*}{$\gamma \gamma$} & $g g \rightarrow h^{0}$ & $a_{g} a_{\gamma}$ & $\begin{array}{l}1.7 \pm 0.6, \text { ATLAS }[43] ; 126.5 \mathrm{GeV} \\
1.4 \pm 0.6, \text { CMS }[41] \\
3.6_{-2.5}^{+3.0}, \text { Tevatron }[40] \\
\text { our average: } 1.6 \pm 0.4\end{array}$ \\
\hline & VBF & $\left(a_{W}+r a_{Z}\right) /(1+r) a_{\gamma}$ & $\begin{array}{l}2.6 \pm 1.3, \text { ATLAS }[43] ; 126.5 \mathrm{GeV} \\
2.1_{-1.1}^{+1.4}, \text { CMS }[41] \\
\text { our average: } 2.3_{-0.9}^{+1.0}\end{array}$ \\
\hline \multirow{3}{*}{$b \bar{b}$} & $W^{*} \rightarrow W h^{0}$ & $a_{W} a_{b}$ & $\begin{array}{l}0.5 \pm 2.2, \text { ATLAS }[1] ; 126 \mathrm{GeV} \\
0.5_{-0.8}^{+0.9}, \text { CMS }[41]\end{array}$ \\
\hline & $Z^{*} \rightarrow Z h^{0}$ & $a_{Z} a_{b}$ & $\begin{array}{l}2.0 \pm 0.7, \text { Tevatron }[40] \\
\text { our average: } 1.4 \pm 0.6\end{array}$ \\
\hline & $t \bar{t} h^{0}$ & $a_{t} a_{b}$ & $-0.8_{-1.9}^{+2.1}, \mathrm{CMS}[41]$ \\
\hline \multirow[t]{3}{*}{$\tau^{+} \tau^{-}$} & $g g \rightarrow h^{0}$ & $a_{g} a_{\tau}$ & $\begin{array}{l}0.4_{-2.0}^{+1.6}, \text { ATLAS }[1] ; 126 \mathrm{GeV} \\
1.3 \pm 1.1, \text { CMS }[41] \\
\text { our average: } 1.0 \pm 0.9\end{array}$ \\
\hline & $\mathrm{VBF}$ & $\left(a_{W}+r a_{Z}\right) /(1+r) a_{\tau}$ & $-1.8_{-0.9}^{+1.0}, \mathrm{CMS}[41]$ \\
\hline & $W^{*} \rightarrow W h^{0}$ & $a_{W} a_{\tau}$ & $0.7_{-3.2}^{+4.1}, \mathrm{CMS}[41]$ \\
\hline
\end{tabular}

Table 1. Combinations of parameters (3rd column) that can be extracted from cross section measurements of various processes. Existing measurements for $M_{h}=125 \mathrm{GeV}$ (except where $M_{h}$ is explicitly specified) are shown in the last column. Our averages do not include any correlations, and are obtained by combining asymmetric errors as in $[44,45]$. 
where $V=W$ or $Z$. For $C_{W}=C_{Z}$, the observable quantity $a_{V}$ can be extracted from the current measurements of $\sigma / \sigma_{\mathrm{SM}}$ for $h^{0}$ production followed by decay into $V V^{*}$ and $\gamma \gamma$ :

$$
a_{V}^{2}=\left(\sigma / \sigma_{\mathrm{SM}}\right)\left(g g \rightarrow h \rightarrow V V^{*}\right) \frac{\left(\sigma / \sigma_{\mathrm{SM}}\right)(\mathrm{VBF} \rightarrow h j j \rightarrow \gamma \gamma j j)}{\left(\sigma / \sigma_{\mathrm{SM}}\right)(g g \rightarrow h \rightarrow \gamma \gamma)}
$$

We assume that the quoted experimental uncertainties correspond to Gaussian distributions, or to bifurcated Gaussian distributions (i.e., two half-Gaussians of same central value glued together) in the case of asymmetric errors. It should be emphasized that this is only a rough approximation, which could be avoided once more information about experimental uncertainties becomes available. With the inputs from table 1, and manipulating the distributions with Monte-Carlo simulations, we find

$$
a_{V}=1.15_{-0.29}^{+0.39}
$$

This implies the following upper limit on the total width:

$$
\Gamma_{h} \leq \Gamma_{h}^{\max }=0.52_{-0.10}^{+0.82} \Gamma_{h}^{\mathrm{SM}} .
$$

Note that, assuming the constraint from $\mathrm{SU}(2)_{W}$ doublets $\left(C_{W}=C_{Z} \equiv C_{V} \leq 1\right), \Gamma_{h}^{\max }$ is a strict upper limit on $\Gamma_{h}$, but the extracted value of $\Gamma_{h}^{\max }$ from current data has an uncertainty; this is indicated at the $1 \sigma$ level in eq. (3.6).

Even though we assumed that the experimental inputs are (bifurcated) Gaussian distributions, the values of $\Gamma_{h}^{\max }$ follow a distribution that is quite different from a bifurcated Gaussian, due to the operations on Gaussians with large variances shown in eqs. (3.2) and (3.4). The $\Gamma_{h}^{\max }$ distribution obtained from current data is shown in figure 1 . The $95 \%$ CL interval for $\Gamma_{h}^{\max } / \Gamma_{h}^{\mathrm{SM}}$ is $0.26-3.56$.

\subsection{Lower limit on $\Gamma_{h}$}

Unlike the above upper limit which relies on a theoretical assumption (upper limit on the $h V V$ couplings), a lower limit on $\Gamma_{h}$ can be derived from the rates required for its observation. The total width of the Higgs boson is given by

$$
\Gamma_{h}=\sum_{\substack{\mathcal{P}=W, Z \\ b, \tau, g, \gamma}} C_{\mathcal{P}}^{2} \Gamma^{\mathrm{SM}}\left(h^{0} \rightarrow \mathcal{P} \mathcal{P}\right)+\Gamma_{X},
$$

where $\Gamma_{X}$ is the $h^{0}$ decay width into final states other than the SM ones. For simplicity, we have not included decays into $Z \gamma, c \bar{c}$ or light-fermion pairs because their sum is at most $3 \%$ of $\Gamma_{h}$ in the SM for any $M_{h}>120 \mathrm{GeV}$. In other words, decays into any of these final states with a width substantially larger than the SM one is included in the exotic width $\Gamma_{X}$.

Given that $\Gamma_{X} \geq 0$, eq. (3.7) implies the following lower limit on the total Higgs width:

$$
\Gamma_{h} \geq \Gamma_{h}^{\mathrm{min}}=\left(\sum_{\substack{\mathcal{P}=W, Z \\ b, \tau, g, \gamma}} a_{\mathcal{P}} \mathcal{B}^{\mathrm{SM}}\left(h^{0} \rightarrow \mathcal{P} \mathcal{P}\right)\right)^{2} \Gamma_{h}^{\mathrm{SM}},
$$




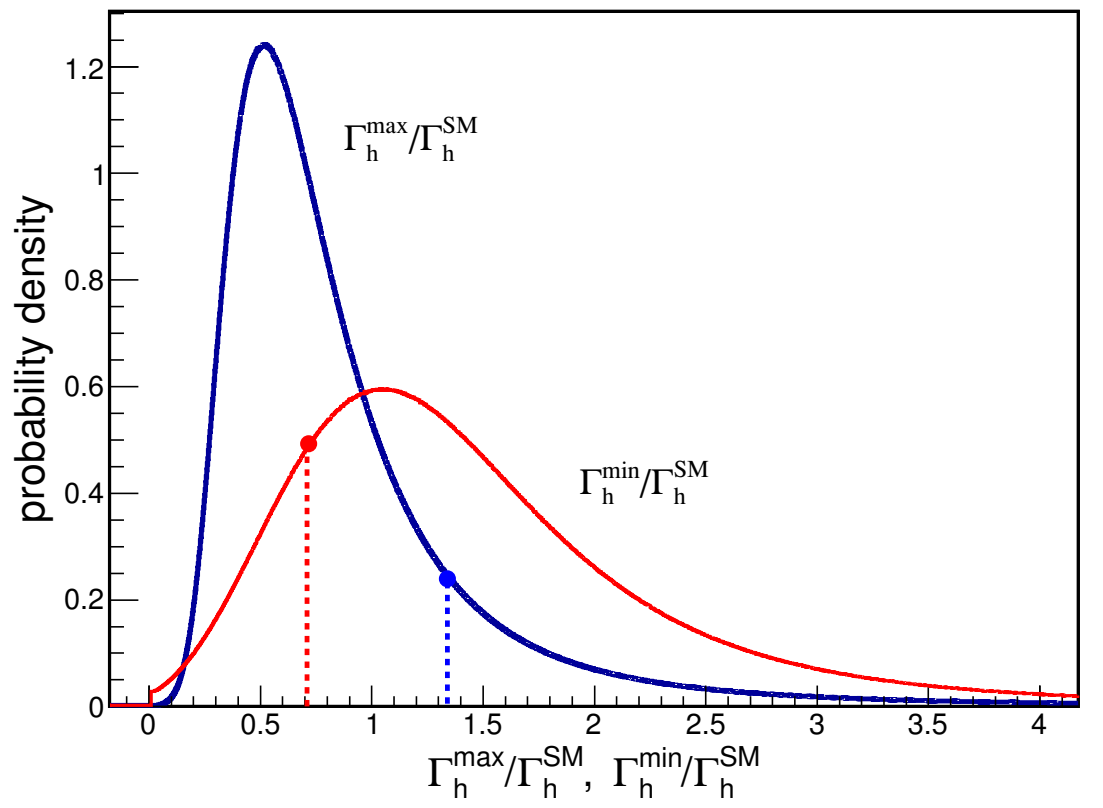

Figure 1. $\Gamma_{h}^{\max }$ distribution obtained from eq. (3.2), and $\Gamma_{h}^{\min }$ distribution obtained from eq. (3.8). The span of $\Gamma_{h} / \Gamma_{h}^{S M}$ lies between the dashed vertical lines, which mark the lower $1 \sigma$ limit on $\Gamma_{h}^{\min }$ and the upper $1 \sigma$ limit on $\Gamma_{h}^{\max }$.

where $\mathcal{B}^{\mathrm{SM}}\left(h^{0} \rightarrow \mathcal{P} \mathcal{P}\right)$ are the theoretically known branching fractions in the SM, and $a_{\mathcal{P}}$ [defined in eq. (2.8)] can be extracted from a fit to the rate measurements. The fact that there is a lower limit on $\Gamma_{h}$ is not surprising given that the observation of a Higgs boson requires a sizable production rate which in turn requires couplings that are not much smaller than the SM ones. However, the exact form of the lower limit was hard to anticipate.

We can extract the distribution for $a_{b}$ from the measurement of the rate for associated production followed by $h^{0} \rightarrow b \bar{b}$ :

$$
\begin{aligned}
a_{b} & =\frac{1}{a_{V}}\left(\sigma / \sigma_{\mathrm{SM}}\right)\left(V h^{0} \rightarrow V b \bar{b}\right) \\
& =1.00_{-0.37}^{+0.91} .
\end{aligned}
$$

The distribution for $a_{g}$ can be obtained from the rate for gluon fusion followed by the $h^{0} \rightarrow W W^{*}, Z Z^{*}$ decays:

$$
\begin{aligned}
a_{g} & =\frac{1}{a_{V}}\left(\sigma / \sigma_{\mathrm{SM}}\right)\left(g g \rightarrow h^{0} \rightarrow V V^{*}\right) \\
& =0.74_{-0.13}^{+0.35} .
\end{aligned}
$$

This then allows the determination of the remaining $a_{\mathcal{P}}$ quantities:

$$
\begin{aligned}
a_{\gamma} & =\frac{1}{a_{g}}\left(\sigma / \sigma_{\mathrm{SM}}\right)\left(g g \rightarrow h^{0} \rightarrow \gamma \gamma\right) \\
& =1.88_{-0.46}^{+0.65} .
\end{aligned}
$$




$$
\begin{aligned}
a_{\tau} & =\frac{1}{a_{g}}\left(\sigma / \sigma_{\mathrm{SM}}\right)\left(g g \rightarrow h^{0} \rightarrow \tau^{+} \tau^{-}\right) \\
& =1.0_{-0.9}^{+1.5} .
\end{aligned}
$$

Note that $a_{\tau}$ could also be extracted from the rate for the VBF process followed by $h^{0} \rightarrow \tau^{+} \tau^{-}$. As can be seen from table 1 , the central value for this rate is about $2 \sigma$ below the predicted value for the case of no Higgs boson. This suggests a large negative fluctuation of the background, so we have chosen not to include this VBF process in a fit until more data is analyzed.

The large uncertainty in $a_{\tau}$ shown in eq. (3.12) raises the issue of what is the meaning of a negative $a_{\mathcal{P}}$. Clearly, negative values for $\sigma / \sigma_{\mathrm{SM}}$ represent downward fluctuations of the background. However, $a_{\mathcal{P}}$ are by definition [see eq. (2.8)] positive quantities, so that it is appropriate to interpret the uncertainties quoted in eqs. (3.9)-(3.12) as distributions with a boundary at the origin. Following the Feldman-Cousins [46] prescription for that case (and assuming approximate Gaussians with variance given by the negative errors), the $1 \sigma$ confidence interval for $a_{\tau}$ becomes $0.3-2.5$. For the purpose of determining the lower limit on $\Gamma_{h}$, it does not make much difference whether we use this interval or the one indicated by eq. (3.12), $0.1-2.5$, because $\mathcal{B}^{\mathrm{SM}}\left(h^{0} \rightarrow \tau^{+} \tau^{-}\right)$is rather small.

For $M_{h}=125 \mathrm{GeV}, \mathcal{B}^{\mathrm{SM}}\left(h^{0} \rightarrow \mathcal{P} \mathcal{P}\right)$ equals $(21.5,2.64,57.7,6.32,8.57,0.228) \%$ for $\mathcal{P}=W, Z, b, \tau, g, \gamma$, respectively, and the total $\mathrm{SM}$ width is $\Gamma_{h}^{\mathrm{SM}}=4.07 \mathrm{MeV}$. The lower limit on $\Gamma_{h}$ can then be computed from eq. (3.8):

$$
\Gamma_{h} \geq \Gamma_{h}^{\min }=1.05_{-0.34}^{+1.26} \Gamma_{h}^{\mathrm{SM}} .
$$

We reiterate that $\Gamma_{h}^{\min }$ is a strict lower limit for $\Gamma_{h}$, but that the value of $\Gamma_{h}^{\min }$ extracted from current data has an uncertainty represented here by the $68.3 \%$ CL range. The $95 \%$ CL interval for $\Gamma_{h}^{\min } / \Gamma_{h}^{\mathrm{SM}}$ is $0.30-4.95$.

Using the upper limit at the $68.3 \% \mathrm{CL}$ for $\Gamma_{h}^{\max }$ given in eq. (3.6), and the lower limit at the $68.3 \%$ CL for $\Gamma_{h}^{\min }$ given in eq. (3.13), we find that the span of the Higgs width is

$$
0.71 \leq \frac{\Gamma_{h}}{\Gamma_{h}^{S M}} \leq 1.34
$$

Note that this span is not a standard confidence interval, because the lower and upper limits arise from separate measurements.

The central value of $\Gamma_{h}^{\max }$ is smaller than that of $\Gamma_{h}^{\min }$. This is not a problem given that both $\Gamma_{h}^{\max }$ and $\Gamma_{h}^{\min }$ are currently rather broad distributions (see figure 1 ), so that the $1 \sigma$ upper limit on $\Gamma_{h}^{\max }$ is larger than the $1 \sigma$ lower limit on $\Gamma_{h}^{\min }$. It is conceivable, though, that more precise future measurements would yield $\Gamma_{h}^{\max }<\Gamma_{h}^{\min }$ at a confidence level of several standard deviations. The likely interpretation of that situation would be that higher $\mathrm{SU}(2)_{W}$ representations have VEVs, so that $\Gamma_{h}^{\max }$ is rescaled by $\left(C_{V}^{\max }\right)^{4}$, with $C_{V}^{\max }>1$.

\section{Limits on Higgs couplings and non-standard decays}

In this section we use the constraints on $\Gamma_{h}$ obtained in the previous section to set an upper limit on the branching fraction $B_{X}$ into exotic final states, and to derive nearly model-independent ranges (which we call spans) for the Higgs couplings. 


\subsection{Upper limit on the exotic branching fraction}

An important implication of the upper limit on $\Gamma_{h}$ is that it leads to an upper limit on the branching fraction for $h^{0}$ decays into non-SM final states, $\mathcal{B}_{X}$. Dividing eq. (3.7) by $\Gamma_{h}$ gives

$$
\mathcal{B}_{X}=1-\frac{1}{\Gamma_{h}} \sum_{\substack{\mathcal{P}=W, Z \\ b, \tau, g, \gamma}} C_{\mathcal{P}}^{2} \Gamma^{\mathrm{SM}}\left(h^{0} \rightarrow \mathcal{P} \mathcal{P}\right) .
$$

Using then the upper limit on $\Gamma_{h}$ given in eqs. (3.1) or (3.2), we find the following upper limit on the branching fraction into exotic final states:

$$
\mathcal{B}_{X} \leq \mathcal{B}_{X}^{\max }=1-\left(\frac{\Gamma_{h}^{\mathrm{SM}}}{\Gamma_{h}^{\max }}\right)^{1 / 2} \sum_{\substack{\mathcal{P}=W, Z, b, \tau, g, \gamma}} a_{\mathcal{P}} \mathcal{B}^{\mathrm{SM}}\left(h^{0} \rightarrow \mathcal{P} \mathcal{P}\right) .
$$

In the case of doublet VEVs $\left(C_{W}=C_{Z} \equiv C_{V}\right)$, the upper limit takes the simpler form

$$
\mathcal{B}_{X}^{\max }=1-a_{V} \sum_{\substack{\mathcal{P}=W, Z, b, \tau, g, \gamma}} a_{\mathcal{P}} \mathcal{B}^{\mathrm{SM}}\left(h^{0} \rightarrow \mathcal{P} \mathcal{P}\right)
$$

The values of $a_{\mathcal{P}}$ given in eqs. (3.5) and (3.9)-(3.12) lead to the following upper limit:

$$
\mathcal{B}_{X} \leq \mathcal{B}_{X}^{\max }=-0.33_{-0.49}^{+0.39} \text {. }
$$

Using the Feldman-Cousins prescription for this manifestly positive observable, we find that the extracted theoretical upper limit is $\mathcal{B}_{X}^{\max }<14 \%$ at the $68.3 \% \mathrm{CL}$ and $\mathcal{B}_{X}^{\max }<47 \%$ at the $95 \%$ CL. For precise measurements of several cross sections, the uncertainties in $a_{\mathcal{P}}$ may become small enough to turn the upper limit on $\mathcal{B}_{X}$ into a severe constraint on physics beyond the SM.

The above limits are derived under the assumption $C_{V}^{\max }=1$. For larger values of $C_{V}^{\max }$ the limits are relaxed. For example, using $C_{V}^{\max } \approx 1.5$ as in the Georgi-Machacek model $[25,26]$ we find $\mathcal{B}_{X}^{\max }<59 \%$ at the $68.3 \%$ CL and $\mathcal{B}_{X}^{\max }<76 \%$ at the $95 \%$ CL.

\subsection{Spans of the Higgs couplings}

From eq. (2.8), we find that the various Higgs couplings discussed in section $2, C_{\mathcal{P}}$ for $\mathcal{P}=W, Z, b, \tau, g, \gamma$, can also be bracketed between lower and upper bounds extracted from current data:

$$
a_{\mathcal{P}}^{1 / 2}\left(\frac{\Gamma_{h}^{\min }}{\Gamma_{h}^{\mathrm{SM}}}\right)^{1 / 4}<C_{\mathcal{P}}<a_{\mathcal{P}}^{1 / 2}\left(\frac{\Gamma_{h}^{\max }}{\Gamma_{h}^{\mathrm{SM}}}\right)^{1 / 4}
$$

Using the distributions for $\Gamma_{h}^{\max }$ (see figure 1), $\Gamma_{h}^{\min }$ and $a_{\mathcal{P}}$, we find the following $68.3 \%$ (95\%) spans for the Higgs couplings:

$$
\begin{array}{rrrr}
(0.74) & 0.97<\left|C_{V}\right| \leq 1, & (0.32) 0.73<\left|C_{b}\right|<1.42(2.34) \\
(0.61) & 0.77<\left|C_{g}\right|<1.07(1.63), & (0.0) 0.3<\left|C_{\tau}\right|<1.4(1.9) \\
\text { (0.92) } 1.19<\left|C_{\gamma}\right|<1.54 \text { (1.93). } &
\end{array}
$$




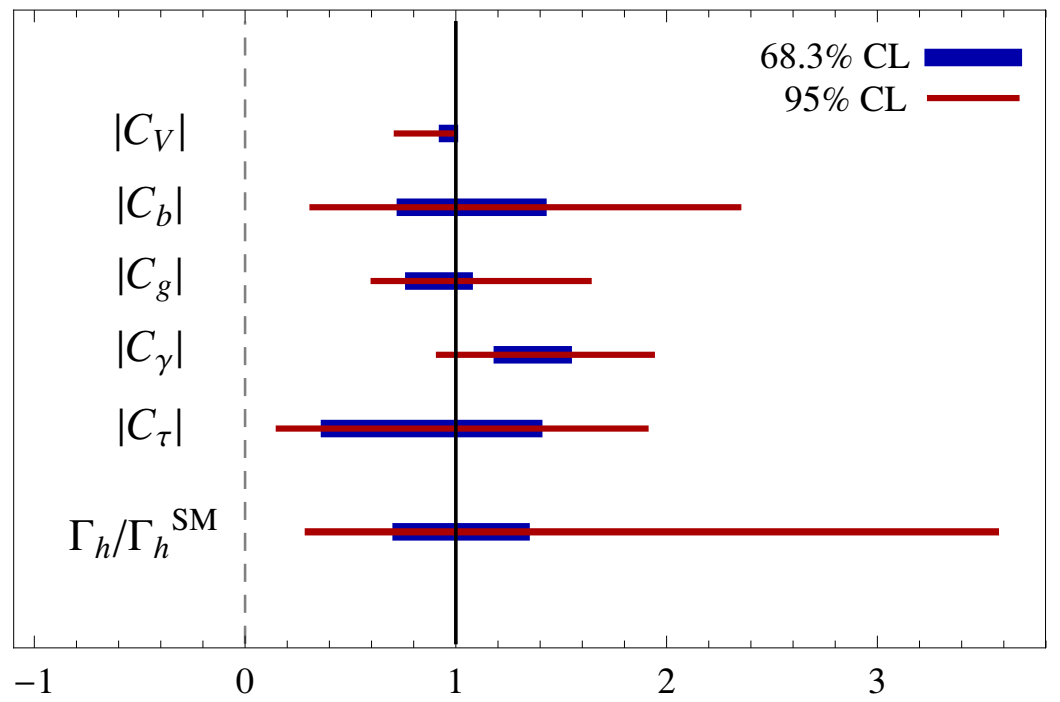

Figure 2. Spans of the Higgs couplings [see eq. (4.5)] and total width, normalized to the SM values. The vertical lines at 0 and 1 correspond to no Higgs boson, and to the SM, respectively. The lefthand edge of each thick (thin) line represents the lower $1 \sigma(95 \%$ CL) point on the distribution for the lower limit, while the right-hand edge represents the upper $1 \sigma$ (95\% CL) point on the distribution for the upper limit (as shown in figure 1 for $\Gamma_{h}$ ). If triplets or higher $\mathrm{SU}(2)_{W}$ representations have VEVs, then the upper limits are pushed to higher values.

These spans arise from the lower limit on $a_{\mathcal{P}}^{1 / 2}\left(\Gamma_{h}^{\min }\right)^{1 / 4}$ and the upper limit on $a_{\mathcal{P}}^{1 / 2}\left(\Gamma_{h}^{\max }\right)^{1 / 4}$, which are obtained from separate experimental inputs, so that they should not be interpreted as standard confidence intervals.

Note that the upper limit on $C_{V}$ is our input based on the assumption that electroweak symmetry breaking is entirely due to the VEVs of doublets. Using the Feldman-Cousins prescription to take into account this prior, we find that the lower limit on $\left|C_{V}\right|$ is relaxed: $\left|C_{V}\right|>0.93(0.72)$ at the $68.3(95) \%$ CL.

The interval for $C_{\tau}$ is the least reliable, given the large uncertainties discussed before eq. (3.5) and after eq. (3.12). Using the Feldman-Cousins prescription for the lower limit $\left|C_{\tau}\right|>0$, this is shifted to 0.16 at the $95 \% \mathrm{CL}$. The fact that the SM value of $C_{\mathcal{P}}=1$ is within the $95 \%$ span for each of the above five couplings is remarkable (see figure 2). Nevertheless, new physics contributions may still have effects larger than $50 \%$ on some of these couplings.

Eq. (3.1) implies that the upper limits on the $C_{\mathcal{P}}$ parameters scale as $C_{V}^{\max }$. The values shown in eq. (4.6) correspond to $C_{V}^{\max }=1$, while $C_{V}^{\max } \approx 1.5$ in models with large triplet VEVs [26]. Even larger values of $C_{V}^{\max }$ are allowed if scalars transforming as 4 of $\mathrm{SU}(2)_{W}$ acquire VEVs [27-29], but models of this type include several charged particles that can be ruled out or discovered at the LHC in the near future. 


\section{Conclusions}

To determine the true nature of the Higgs-like resonance discovered by the ATLAS and CMS experiments, we need precise determinations of its underlying couplings to SM particles, extracted without making unnecessary theoretical assumptions. The impossibility of measuring $\Gamma_{h}$ at the LHC therefore poses a significant problem, in addition to masking whether the new resonance has exotic decays that may be difficult to observe experimentally. We have taken a novel approach to this problem, by observing that $\Gamma_{h}$ has a model-independent lower bound, and an upper bound that relies only on the weak assumption that the Higgs-like couplings to $W W$ and $Z Z$ is not larger than (or not much larger than) the SM values. We showed that $\Gamma_{h}^{\min }$ and $\Gamma_{h}^{\max }$ can be extracted separately from data for different combinations of Higgs-like signal strengths. This allows to confine $\Gamma_{h}$ itself to a certain range between lower and upper limits; this span is not a standard confidence interval, but the limits themselves, being extracted from data, have $68.3 \%$ and 95\% CL intervals that we have estimated. It is nontrivial that the resulting span for $\Gamma_{h}$ is approximately centered on the SM value.

This same information can then be propagated to both an upper limit on the Higgs exotic branching fraction, and pairs of lower and upper limits for various Higgs couplings to SM gauge bosons and fermions. For the exotic branching fraction the extracted value of the upper bound is $14 \%$ at $68.3 \%$ CL in the underlying data, and $47 \%$ at $95 \%$ CL. For the Higgs couplings we find the spans displayed in figure 2. At 95\% CL in the extracted limits all of these spans include the SM value. Notice however that by far the largest uncertainty in the current extraction of limits applies to the determination of $\Gamma_{h}^{\min }$ and $\Gamma_{h}^{\max }$ themselves.

With additional data the methodology described here will give increasingly important constraints on the properties of the newly discovered particle, and is complementary to other approaches currently being pursued. The major shortcoming of our analysis is our ignorance of the details of the experimental uncertainties in the published data; this can be overcome easily if the experimental collaborations themselves perform the analysis that we are advocating.

Open Access. This article is distributed under the terms of the Creative Commons Attribution License which permits any use, distribution and reproduction in any medium, provided the original author(s) and source are credited.

\section{References}

[1] ATLAS collaboration, Observation of a new particle in the search for the standard model Higgs boson with the ATLAS detector at the LHC, Phys. Lett. B 716 (2012) 1 [arXiv: 1207.7214] [INSPIRE].

[2] CMS collaboration, Observation of a new boson at a mass of $125 \mathrm{GeV}$ with the CMS experiment at the LHC, Phys. Lett. B $\mathbf{7 1 6}$ (2012) 30 [arXiv:1207.7235] [INSPIRE].

[3] A. Djouadi, The anatomy of electro-weak symmetry breaking. I: the Higgs boson in the standard model, Phys. Rept. 457 (2008) 1 [hep-ph/0503172] [INSPIRE]. 
[4] B.A. Dobrescu, G.L. Landsberg and K.T. Matchev, Higgs boson decays to CP odd scalars at the Tevatron and beyond, Phys. Rev. D 63 (2001) 075003 [hep-ph/0005308] [InSPIRE].

[5] C.-R. Chen, M.M. Nojiri and W. Sreethawong, Search for the elusive Higgs boson using jet structure at LHC, JHEP 11 (2010) 012 [arXiv:1006.1151] [INSPIRE].

[6] A. Falkowski, D. Krohn, L.-T. Wang, J. Shelton and A. Thalapillil, Unburied Higgs boson: jet substructure techniques for searching for Higgs' decay into gluons,

Phys. Rev. D 84 (2011) 074022 [arXiv:1006.1650] [inSPIRE].

[7] D. Zeppenfeld, R. Kinnunen, A. Nikitenko and E. Richter-Was, Measuring Higgs boson couplings at the CERN LHC, Phys. Rev. D 62 (2000) 013009 [hep-ph/0002036] [INSPIRE].

[8] D. Zeppenfeld, Higgs couplings at the LHC, eConf C 010630 (2001) P123 [hep-ph/0203123] [INSPIRE].

[9] M. Dührssen et al., Determination of Higgs-boson couplings at the LHC, hep-ph/0407190 [INSPIRE].

[10] D. Carmi, A. Falkowski, E. Kuflik and T. Volansky, Interpreting LHC Higgs results from natural new physics perspective, JHEP 07 (2012) 136 [arXiv:1202.3144] [INSPIRE].

[11] A. Azatov, R. Contino and J. Galloway, Model-independent bounds on a light Higgs, JHEP 04 (2012) 127 [arXiv: 1202.3415] [INSPIRE].

[12] M. Klute, R. Lafaye, T. Plehn, M. Rauch and D. Zerwas, Measuring Higgs couplings from LHC data, Phys. Rev. Lett. 109 (2012) 101801 [arXiv:1205.2699] [INSPIRE].

[13] F. Bonnet, T. Ota, M. Rauch and W. Winter, Interpretation of precision tests in the Higgs sector in terms of physics beyond the standard model, Phys. Rev. D 86 (2012) 093014 [arXiv: 1207.4599] [INSPIRE].

[14] M.E. Peskin, Comparison of LHC and ILC capabilities for Higgs boson coupling measurements, arXiv:1207.2516 [INSPIRE].

[15] D. Carmi, A. Falkowski, E. Kuflik, T. Volansky and J. Zupan, Higgs after the discovery: a status report, JHEP 10 (2012) 196 [arXiv:1207.1718] [INSPIRE].

[16] C. Englert, M. Spannowsky and C. Wymant, Partially (in)visible Higgs decays at the LHC, Phys. Lett. B 718 (2012) 538 [arXiv:1209.0494] [INSPIRE].

[17] R. Lafaye, T. Plehn, M. Rauch, D. Zerwas and M. Dührssen, Measuring the Higgs sector, JHEP 08 (2009) 009 [arXiv:0904.3866] [INSPIRE].

[18] V. Barger, M. Ishida and W.-Y. Keung, Total width of $125 \mathrm{GeV}$ Higgs boson, Phys. Rev. Lett. 108 (2012) 261801 [arXiv:1203.3456] [INSPIRE].

[19] P.P. Giardino, K. Kannike, M. Raidal and A. Strumia, Is the resonance at $125 \mathrm{GeV}$ the Higgs boson?, Phys. Lett. B 718 (2012) 469 [arXiv:1207.1347] [INSPIRE].

[20] P.P. Giardino, K. Kannike, M. Raidal and A. Strumia, Reconstructing Higgs boson properties from the LHC and Tevatron data, JHEP 06 (2012) 117 [arXiv: 1203.4254] [INSPIRE].

[21] J. Espinosa, C. Grojean, M. Muhlleitner and M. Trott, First glimpses at Higgs' face, JHEP 12 (2012) 045 [arXiv:1207.1717] [INSPIRE].

[22] J.R. Espinosa, M. Muhlleitner, C. Grojean and M. Trott, Probing for invisible Higgs decays with global fits, JHEP 09 (2012) 126 [arXiv:1205.6790] [INSPIRE]. 
[23] S. Banerjee, S. Mukhopadhyay and B. Mukhopadhyaya, New Higgs interactions and recent data from the LHC and the Tevatron, JHEP 10 (2012) 062 [arXiv:1207.3588] [INSPIRE].

[24] LHC Higgs Cross section Working Group collaboration, A. David et al., LHC HXSWG interim recommendations to explore the coupling structure of a Higgs-like particle, arXiv: 1209.0040 [INSPIRE].

[25] H. Georgi and M. Machacek, Doubly charged Higgs bosons, Nucl. Phys. B 262 (1985) 463 [INSPIRE].

[26] H.E. Logan and M.-A. Roy, Higgs couplings in a model with triplets, Phys. Rev. D 82 (2010) 115011 [arXiv: 1008.4869] [INSPIRE].

[27] I. Low and J. Lykken, Revealing the electroweak properties of a new scalar resonance, JHEP 10 (2010) 053 [arXiv: 1005.0872] [INSPIRE].

[28] S. Chang, C.A. Newby, N. Raj and C. Wanotayaroj, Revisiting theories with enhanced Higgs couplings to weak gauge bosons, Phys. Rev. D 86 (2012) 095015 [arXiv:1207.0493] [INSPIRE].

[29] I. Low, J. Lykken and G. Shaughnessy, Have we observed the Higgs (imposter)?, Phys. Rev. D 86 (2012) 093012 [arXiv:1207.1093] [INSPIRE].

[30] A. Falkowski, S. Rychkov and A. Urbano, What if the Higgs couplings to $W$ and $Z$ bosons are larger than in the standard model?, JHEP 04 (2012) 073 [arXiv:1202.1532] [INSPIRE].

[31] CDF and DØ collaborations, Evidence for a particle produced in association with weak bosons and decaying to a bottom-antibottom quark pair in Higgs boson searches at the Tevatron, Phys. Rev. Lett. 109 (2012) 071804 [arXiv:1207.6436] [InSPIRE].

[32] G. Pocsik and G. Zsigmond, Decays of heavy Higgs bosons into vector bosons, Phys. Lett. B 112 (1982) 157 [INSPIRE].

[33] H.E. Haber and H.E. Logan, Radiative corrections to the Z $b \bar{b}$ vertex and constraints on extended Higgs sectors, Phys. Rev. D 62 (2000) 015011 [hep-ph/9909335] [INSPIRE].

[34] C.-W. Chiang, T. Nomura and K. Tsumura, Search for doubly charged Higgs bosons using the same-sign diboson mode at the LHC, Phys. Rev. D 85 (2012) 095023 [arXiv:1202.2014] [INSPIRE].

[35] Y. Bai, J. Fan and J.L. Hewett, Hiding a heavy Higgs boson at the $7 \mathrm{TeV} L H C$, JHEP 08 (2012) 014 [arXiv:1112.1964] [INSPIRE].

[36] B.A. Dobrescu, G.D. Kribs and A. Martin, Higgs underproduction at the LHC, Phys. Rev. D 85 (2012) 074031 [arXiv:1112.2208] [INSPIRE].

[37] B. Batell, S. Gori and L.-T. Wang, Exploring the Higgs portal with $10 \mathrm{fb}^{-1}$ at the LHC, JHEP 06 (2012) 172 [arXiv:1112.5180] [INSPIRE].

[38] K. Kumar, R. Vega-Morales and F. Yu, Effects from new colored states and the Higgs portal on gluon fusion and Higgs decays, Phys. Rev. D 86 (2012) 113002 [arXiv:1205.4244] [INSPIRE].

[39] T. Han, G. Valencia and S. Willenbrock, Structure function approach to vector boson scattering in pp collisions, Phys. Rev. Lett. 69 (1992) 3274 [hep-ph/9206246] [INSPIRE].

[40] Tevatron New Physics Higgs Working Group, CDF and D $\varnothing$ collaborations, Updated combination of $C D F$ and $D \varnothing$ searches for standard model Higgs boson production with up to $10.0 \mathrm{fb}^{-1}$ of data, arXiv:1207.0449 [INSPIRE]. 
[41] CMS collaboration, Observation of a new boson with a mass near $125 \mathrm{GeV}$, CMS-HIG-12-020, CERN, Geneva Switzerland July 2012.

[42] ATLAS collaboration, Observation of an excess of events in the search for the standard model Higgs boson in the $H \rightarrow Z Z^{(*)} \rightarrow 4 \ell$ channel with the ATLAS detector, ATLAS-CONF-2012-092, CERN, Geneva Switzerland July 2012.

[43] ATLAS collaboration, Observation of an excess of events in the search for the standard model Higgs boson in the gamma-gamma channel with the ATLAS detector, ATLAS-CONF-2012-091, CERN, Geneva Switzerland July 2012.

[44] R. Barlow, Asymmetric statistical errors, physics/0406120 [INSPIRE].

[45] Asymmetric statistical errors webpage, http://www.slac.stanford.edu/ barlow/java/statistics5.html.

[46] G.J. Feldman and R.D. Cousins, A unified approach to the classical statistical analysis of small signals, Phys. Rev. D 57 (1998) 3873 [physics/9711021] [INSPIRE]. 